\title{
AUTOESTIMA Y SU INFLUENCIA EN LA CULTURA ORGANIZACIONAL DE LOS DOCENTES
}

\section{SELF-ESTEEM AND ITS INFLUENCE ON THE ORGANIZATIONAL CULTURE OF TEACHERS}

\section{DOI: https://doi.org/10.53595/rlo.v1.i2.010}

\author{
Yaneth Adelaida Ticona Ancco \\ Universidad Nacional del Altiplano \\ https://orcid.org/0000-0003-4413-8012 \\ yanethancco@gmail.com
}

\author{
Elisa Candida Garavito Checalla \\ Universidad Nacional del Altiplano \\ https://orcid.org/0000-0003-1078-8578 \\ egaravito@unap.edu.pe
}

\section{RESUMEN}

Hoy en día en nuestro país, las instituciones educativas se ven envueltas en asumir nuevos retos en la organización, en concordancia con la propia autoestima de sus maestros; la investigación tiene el objetivo general de determinar la influencia del nivel de autoestima de los docentes en la cultura organizacional de las instituciones educativas primarias (IEP) red Aymaras de Chucuito en el año 2019, la metodología utilizada es descriptiva explicativa con un cuestionario como instrumento, aplicado a una muestra de 26 docentes, en cuanto a los resultados se puede observar que en la escala de Autoestima Alta se tiene un $73.1 \%$ de docentes, seguido de un $26.9 \%$ con Autoestima Media y ningún Docente en la escala de Autoestima Baja, destacando que el nivel de autoestima tiene como dimensiones: física, social, afectica, académica y ética; en lo relacionado a la cultura organizacional se observó una alta cultura organizacional en un $42.3 \%$ de docentes, seguido de un $38.5 \%$ de ellos con cultura organizacional media y un $19.2 \%$ con baja cultura organizacional, teniendo en cuenta las dimensiones: innovación y toma de riesgo, atención al detalle, orientación a los resultados, orientación a las personas, orientación al equipo, energía y estabilidad. Se concluye que: existe una influencia significativa de la variable autoestima en la cultura organizacional en los docentes de las IEP Red Aymaras de Chucuito en el año 2019.

Palabras clave: Autoestima, cultura, energía, estabilidad, innovación, organización, red educativa.

\section{ABSTRACT}

Today in our country, educational institutions are involved in assuming new challenges in the organization, in accordance with the self-esteem of their teachers; The research has the general objective of determining the influence of the level of self-esteem of teachers in the organizational culture of primary educational institutions (IEP) Aymaras de Chucuito network in 2019, the methodology used is descriptive explanatory with a questionnaire as an instrument, applied to a sample of 26 teachers, regarding the results 
it can be observed that in the High Self-Esteem scale there are $73.1 \%$ of teachers, followed by $26.9 \%$ with Medium Self-esteem and no Teacher in the Low Self-esteem scale, highlighting that the level of self-esteem has as dimensions: physical, social, affective, academic and ethical; Regarding organizational culture, a high organizational culture was observed in $42.3 \%$ of teachers, followed by $38.5 \%$ of them with medium organizational culture and $19.2 \%$ with low organizational culture, taking into account the dimensions: innovation and decision making. risk, attention to detail, results orientation, people orientation, team orientation, energy and stability. It is concluded that: there is a significant influence of the self-esteem variable in the organizational culture in the teachers of the IEP Red Aymaras de Chucuito in the year 2019.

Keywords: Culture, educational network, energy, innovation, organization, selfesteem, stability.

\section{INTRODUCCIÓN}

El desempeño docente y la autoestima, son aspectos muy imprescindibles y valiosos en todos los ámbitos, desde lo social hasta educacional. Para Valero-Ancco et al.(2021) el estado promover una alta identidad profesional en los profesores, para un mejor desempeño y aprendizaje en los estudiantes. Estimulando las condiciones en las que labora, brindándole recursos y oportunidades de crecimiento profesional (MartínezTorres 2020). Cuando se habla de autoestima, se entiende que es una variación constante de grados, los cuales van de un polo a otro, y entre esos parámetros, cabe un conjunto de posibilidades de ubicación (Canazas Arizaca 2020). Para Aðalsteinsson et al. (2014), un autoestima alta, guarda relación con adaptaciones exitosas a eventos que se presentan en la vida del individuo. Una baja autoestima puede generar efectos negativos en la calidad de enseñanza que reciben los estudiantes, su rendimiento académico, el bienestar social y el clima escolar (Khezerlou 2017). Con respecto al autoestima de los docentes, ellos deben lograr un desarrollo profesional y personal, reflejado en la manera en que desarrolla sus tareas y las relaciones que establece con su entorno (Hernández 2017). Para Pineda et al (2019), la autoestima docente, es la apreciación de su propia valía, la cual emplean para

llevar a cabo una reflexión, aceptar y tomar decisiones sobre su vida personal y profesional. Wilhelm et al. (2012) señalan que, es una competencia profesional de carácter socioafectiva del profesor la cual se expresa en los distintos procesos educativos. García (2012) complementa lo dicho anteriormente, afirmando que esta competencia emocional, va enlazada a la autovaloración.

El profesionalismo de un educador se encuentra conformada por conocimientos y habilidades que intervienen en el desempeño eficiente de la actividad pedagógica (Zegarra \& Velázquez 2016). A lo largo de su carrera, García (2012), logró observar que los profesores tienden a tener un bajo autoconcepto de ellos mismos; dado que suelen 
compararse con los estándares de excelencia o perfección (Tabassum 2012). De acuerdo con (Sebastian 2012), el docente necesita llevar a cabo una reflexión, aceptarse y asumir en su propia dinámica vivencial y profesional. La percepción que posee el docente de sí mismo, como comunicador juega un papel importante en su autoconcepto, dado que la enseñanza no será eficaz sin una comunicación pedagógica que sea de alta calidad (Glotova \& Wilhelm 2014). Para Peñaherrera León et al.(2014), el desarrollo personal y profesional, la actitud, motivación y estímulos externos son los elementos que configuran el autoestima de un profesor y por otro lado, él transmite su situación anímica a sus alumnos (Sebastian 2012). Problemas físicos, trastornos psicológicos, cambios en el comportamiento y adicciones son las maneras en que expresa su malestar un profesor (Sadeghi \& Sa'adatpourvahid 2016), especialmente cuando las ocupaciones excedan los recursos al momento de enseñar (Aldrete et al. 2011). De acuerdo con Wilhelm et al. (2012), se debe tomar conciencia de la valía del sujeto, ya que es clave en la adquisición del fundamento cultural que sustenta su valía.

En lo que respecta a la Cultura organizacional, repercute sobre las motivaciones y el comportamiento de los miembros de la institución, teniendo su origen en la sociología (Iglesias \& Sánchez 2015). Tinoco Gómez et al. (2014), señala que la cultura es el modo particular de vida de una organización, como conjunto social de ideas compartidas, las cuales son matizadas de manera subjetiva por una escala de valores que la colectividad, asigna de una manera particular (González Díaz et al. 2016). La cultura organizacional, es considerada como uno de los pilares de la competitividad de las organizaciones o empresas (Moreno \& Pineda 2019). Esta se encontraría conformada por las ideas que tienen sus colaboradores (Llancari Morales \& Alania Contreras 2020). La cultura interviene en el proceso de modificación de la organización (Ferrer 2015), una de las características de la cultura organizacional, es que, es aprendida y es una respuesta al entorno (Rodríguez Castellanos \& Romo Rojas 2013). El contexto organizacional influye en el liderazgo transformacional y sus progresos (Vasquéz Alatorre 2013), se basan en ciertos elementos que se encargan de orientar las actitudes y el comportamiento sus integrantes (Loyo De Falcón 2015). Para Bernal et al.(2015) cuando la organización no guarda coherencia entre su sistema de creencias y sus operaciones, desencadena en los grupos que la integran actitudes de conformidad, de autoprotección o conductas negativas hacia la institución (Ramírez Campos \& Domínguez Aguirre 2012). La cultura organizacional dota de valores culturales a los miembros del centro educativo (Rahmadani \& Hanum 2020), esta figura, según Hamui-Sutton et al. (2014), alude a un 
modo de vida, una manera particular de interacción, creencias, valores y expectativas que se tienen

El objetivo general de la presente investigación, determinar la influencia del nivel de autoestima de los docentes en la cultura organizacional de la I.E.P red Aymaras de Chucuito, 2019. Teniendo como hipótesis, el nivel de autoestima de los docentes influye significativamente en la cultura organizacional de la I.E.P red Aymaras de Chucuito, 2019.

\section{MATERIALES Y MÉTODOS}

\section{Ámbito o lugar de estudio}

La investigación se ejecutó en las instituciones educativas primarias red aymaras de Chucuito, 2019, ubicado en la provincia de Puno, distrito Chucuito correspondiente a la UGEL Puno. Es una investigación cuantitativa, de tipo descriptivo explicativo, donde se utilizó el método empírico de la observación, de diseño no probabilístico por conveniencia.

\section{Descripción de métodos}

La población estuvo constituida por los docentes de las Escuelas Primarias de la red Aymaras de Chucuito de la Ugel Puno, la muestra fue de tipo no probabilístico, aplicando una técnica de muestreo intencional o por conveniencia, tomando una cantidad representativa de la población, 36 docentes, (06) varones y (20) mujeres, de la I.E.P. Red Aymaras de Chucuito, 2019. Las variables analizadas para la siguiente investigación, serán: la variable independiente Autoestima y la variable dependiente, la Cultura Organizacional

Se utilizó la técnica de la encuesta para la evaluación de las dos variables, que consiste en indagar la opinión que tiene la población sobre el tema de estudio. Los instrumentos utilizados fueron los cuestionarios. Se utilizó el paquete estadístico SPSS para realizar la prueba estadística de la Chi cuadrada de Pearson, dado que el diseño de investigación es descriptivo explicativo y esta prueba estadística, permite ver la influencia del nivel de autoestima de los docentes en la cultura organizacional.

\section{RESULTADOS Y DISCUSIÓN}

\section{Nivel de autoestima de los docentes de las I.E.P red Aymaras de Chucuito, 2019}

Tomando en cuenta la escala cualitativa del presente trabajo; se puede observar que en la escala de Autoestima Alta se tiene un 73,1\% que representa a 19 docentes, seguido de un 
26,9\% que representa a 7 docentes que se encuentran en escala de Autoestima Media y ningún docente en la escala de Autoestima Baja.

Tabla 1

Resultados del nivel de autoestima promedio de los docentes de las I.E.P Red Aymaras de Chucuito,

\begin{tabular}{lll}
\multicolumn{3}{c}{2019} \\
Escala & $\mathbf{N}$ & $\mathbf{\%}$ \\
\hline Autoestima alta & 19 & $73,1 \%$ \\
Autoestima media & 7 & $26,9 \%$ \\
Autoestima baja & 0 & $0,0 \%$ \\
Total & 26 & $100,0 \%$ \\
\hline
\end{tabular}

Este promedio mostrado nos da a conocer de una mejor manera el nivel de autoestima por parte de los profesores, información importante en este tipo de estudios dato, tal como indica (Naranjo 2007), que existe una valoración global en las personas cuando se trata de apreciarse a sí mismo, y de esta manera sentirse amado y valorado por los demás. Estos resultados se contrastan con los de Costilla Castillo (2015) que encontró a un 77.4\% de los docentes encuestados con un nivel alto de autoestima y un $22.6 \%$ con un nivel medio. Asimismo Cáceres (2017) que halló una autoestima elevada en $61.7 \%$ de los docentes encuestados, lo mismo que comparte Cruz (2017) que halló en 55.2\% una autoestima alta y a $29.3 \%$ docentes con autoestima media baja

A sí mismo, los docentes al poseer una autoestima alta (Panesso \& Arango 2017), indica que su comportamiento singular es como compartir cosas y hechos, ser agradecido, ser reflexivo, ser respetuoso con sus congéneres, ser de mente abierta a lo nuevo, actuar de manera honesta de manera responsable, ser comprensible, se siente importante, tiene confianza en sí mismo

\section{Cultura organizacional de la I.E.P. red Aymaras de Chucuito, 2019}

La tabla 2 muestra los resultados que pertenecen a la cultura organizacional de los docentes de las I.E.P Red Aymaras de Chucuito, 2019.

Tabla 2

Resultados de la cultura organizacional de los docentes de las I.E.P Red Aymaras de Chucuito, 2019

\begin{tabular}{lll}
\hline Escala & $\mathbf{N}$ & $\mathbf{\%}$ \\
\hline Alta & 11 & $42,3 \%$ \\
Media & 10 & $38,5 \%$ \\
Baja & 5 & $19,2 \%$ \\
Total & 26 & $100,0 \%$ \\
\hline
\end{tabular}

Tomando en cuenta la escala cualitativa del presente trabajo; se puede observar que tienen una alta cultura organizacional a un $42,3 \%$ que representa a 11 docentes, seguido de un 
$38,5 \%$ que representa a 10 docentes que tienen en medida media cultura organizacional y un $19,2 \%$ que representa a 5 docentes es baja su cultura organizacional.

Estos resultados se comparan con los de Romero (2019), que encuentra un nivel alto de cultura organizacional en $11.4 \%$ de los docentes encuestados y un $80 \%$ de ellos con un nivel medio de dicha variable. Asimismo, Barboza Montes (2018) que halló un nivel regular de $45.8 \%$ en la cultura organizacional de los docentes de una institución educativa y Lacherre Calderón (2017) que halló a un 93\% de docentes con un nivel alto de cultura organizacional, mientras que solo el $7 \%$ tenía un nivel medio y ninguno tuvo un nivel bajo.

Este resultado nos da una idea clara acerca de la variable cultura organizacional en forma global en sus diferentes dimensiones que lo conforman, como manifiesta Figueroa (2015), la Cultura Organizacional son elementos que se relacionan dinámicamente a través de actividades con la finalidad de dar cumplimiento a los objetivos y metas planificadas. La cultura organizacional en la actualidad tiene gran importancia, dado que determina la manera en cómo funciona una organización o empresa, y esto se refleja principalmente en las estrategias, en las estructuras y en los sistemas.

\section{Influencia del nivel de autoestima de los docentes en la cultura organizacional de la}

\section{I.E.P red Aymaras de Chucuito, 2019}

A continuación, se muestra los resultados de las dos variables en estudio, con el fin de hallar la influencia del nivel de autoestima en la cultura organizacional.

Representados con "a" para la variable Nivel de Autoestima y "b" para el la Cultura Organizacional de los Docentes de las I.E.P. Red Aymaras de Chucuiito, 2019; del cuadro se desprende los resultados que se reemplazara a la fórmula planteada.

$$
\chi_{o}^{2}=\sum_{j=1}^{b} \sum_{i=1}^{a} \frac{\left(O_{i j}-E_{i j}\right)^{2}}{E_{i j}} \rightarrow \chi_{k}^{2}
$$

Dónde : $\quad \mathrm{K}=(\mathrm{a}-1)(\mathrm{b}-1)$

$\mathrm{a}=$ Dimensiones de la variable autoestima (variable independiente)

$\mathrm{b}=$ Dimensiones de la variable cultura organizacional (variable dependiente)

Además: $\quad \mathrm{O}_{\mathrm{ij}}$ : son valores observados.

$\mathrm{E}_{\mathrm{ij}}$ : son valores esperados.

$E_{i j}=\underline{f}_{\text {. }} . f_{j}$ son los valores esperados en cada $n$ casilla de la tabla. 
Los valores calculados en la tabla 3 , hacen posible que se acepte la hipótesis alternativa, rechazando la hipótesis nula; esto quiere decir que existe una influencia significativa de la autoestima en la cultura organizacional de los docentes de las instituciones educativas primarias red Aymaras de Chucuito en el año 2019, estos resultados permitieron el logro del objetivo general de la investigación.

Tabla 3

Frecuencias observadas de la autoestima y la cultura organizacional

\begin{tabular}{lccccc}
\hline \multicolumn{1}{c}{ Autoestima } & \multicolumn{2}{c}{ Cultura organizacional } & & \\
& Siempre & casi siempre & A veces & nunca & Total \\
\hline Muy de acuerdo & 3 & 3 & 1 & 0 & 7 \\
De acuerdo & 1,88 & 1,15 & 0,27 & 0,00 & 0 \\
En descuerdo & 3 & 5 & 2 & 0 & 10 \\
Muy en desacuerdo & 2,69 & 1,92 & 0,54 & 0,00 & 8 \\
& 2,15 & 2 & 4,08 & 0,08 & 1 \\
Total & 0 & 0,77 & 0 & 1 & 0,08 \\
\hline
\end{tabular}

1) Cálculo de valor de Chi cuadrada: con los datos de la tabla 3 se procedió a calcular el valor de chi cuadrado calculado el cual fue : $\mathrm{X}^{2}$ calculado $=47,198$. De la misma manera el chi calculado crítico se extrae de la tabla estadística, cuyo valor es $X^{2}$ crítico $=16,92$

2) Nivel de significancia: $\alpha=0,05$

\section{3) Regla de Decisión:}

$X^{2}$ calculada $>X^{2}$ crítico se rechaza la Ho y se acepta la Ha

$\mathrm{X}^{2}$ calculada $<\mathrm{X}^{2}$ crítico se rechaza la Ha y se acepta la Ho

Como X2 calculado $=47198$ es mayo que X2 crítico $=16,92$, se rechaza la Ho y se acepta la Ha.

Después de realizar y analizar la interpretación de los resultados a los que se ha arribado en la presente investigación podemos indicar que, existe influencia del nivel de autoestima en la cultura organizacional, resultados muy parecidos son los que encontró Canazas Arizaca (2020), quien también manifestó en sus resultados que la autoestima influye en la cultura organizacional de los docentes; así mismo E. Irigoyen Rivera et al. (2011), sostiene las mismas conclusiones hallando un coeficiente de Pearson de $\mathrm{r}=0.85$; y por último se menciona la investigación de Pacheco (2019), cuyos resultados indican que existe relación significativa entre autoestima y clima organizacional, consiguiendo 
un valor $\mathrm{r}=0.687$ en su prueba de Pearson, lo mismo que comparte Loli Pineda y Cuba B. (2007) al encontrar un valor de 0.544 con la prueba de Spearman. Lo que nos lleva finalmente a inferir que la autoestima afecta directamente en mayor o menor grado a otras variables propias de la gestión de una institución, ya sea cultura organizacional, clima organizacional entre otros.

\section{CONCLUSIONES}

Existe una influencia significativa de la autoestima en la cultura organizacional de los docentes de las I.E.P Red Aymaras de Chucuito en el año 2019, según el valor del chi cuadrado calculado con los datos de la tabla 3 cuyo valor es de 47,198. Así mismo el valor de chi cuadrado crítico cuyo valor es de 16,92 permite deducir que existe esta influencia altamente significativa. En cuanto al nivel de autoestima de los docentes, se pudo observar que en la escala de Autoestima Alta se tiene un $73,1 \%$ seguido de un $26,9 \%$ que se encuentran en escala de Autoestima Media y ningún docente en la escala de Autoestima Baja. En lo relacionado a la cultura organizacional teniendo en cuenta la escala cualitativa del presente trabajo; se pudo observar que tienen una alta cultura organizacional un $42,3 \%$ de docentes, seguido de un $38,5 \%$ que tienen cultura organizacional media y un 19,2\% con baja cultura organizacional.

\section{AGRADECIMIENTOS}

A los directores y docentes de la red Aymaras de Chucuito, por el apoyo brindado.

\section{CONFLICTO DE INTERÉS}

Las autoras (YATA; ECGC), no tienen conflictos de intereses de ninguna índole.

\section{REFERENCIAS}

Aðalsteinsson, R. I., Frímannsdóttir, I. B., \& Islandia, U. De. (2014). Profesores, autoestima y autoeficacia. Revista Escandinava de Investigacón Educativa, 58, 540-550. https://doi.org/http://dx.doi.org/10.1080/00313831.2013.773559

Aldrete, M., Aranda, C., Valencia, S., \& Salazar, J. (2011). Satisfacción laboral y síndrome burnout en docentes de secundaria. Revista de Educación y Desarrollo, 15-22. https://www.cucs.udg.mx/revistas/edu_desarrollo/anteriores/17/017_Aldrete.pdf

Barboza Montes, W. J. (2018). Cultura organizacional y desempeño docente en la institución educativa primaria $N^{o} 80392$ Andrés Salvador Díaz Sagástegui. Chepén - 2017 [Universidad César Vallejo]. https://repositorio.ucv.edu.pe/bitstream/handle/20.500.12692/11835/barboza_mw.pdf?sequence=1 \&isAllowed $=\mathrm{y}$

Bernal, I., Pedraza Melo, N. A., \& Sánchez Limón, M. L. (2015). El clima organizacional y su relación con la calidad de los servicios públicos de salud: diseño de un modelo teórico. Estudios Gerenciales, 31(134), 8-19. https://doi.org/http://dx.doi.org/10.1016/j.estger.2014.08.003

Cáceres, R. (2017). Autoestima y desempeño laboral docente en la institución educativa secundaria "Nuestra Señora de Alta Gracia", Ayaviri 2016 [Universidad Nacional del Altiplano]. http://repositorio.unap.edu.pe/bitstream/handle/UNAP/6228/EPG891-00891-

01.pdf?sequence $=1 \&$ isAllowed $=y$

Canazas Arizaca, G. (2020). Influencia de autoestima en la cultura organizacional de los docentes de la escuela profesional de administración y negocios internaiconales de la facultad ciencias administrativas de la universidad Andina Néstor Cáceres Velásquez de Juliaca. Revista de 
Investigaciones de La Escuela de Posgrado de La Universidad Nacional Del Altiplano, 9(1), 14731480. https://doi.org/DOI: http://dx.doi.org/10.26788/riepg.2020.1.164

Costilla Castillo, P. C. (2015). Autoestima en los docentes de la Institución Educativa Particular Sarita Colonia, distrito Callao, 2017 [Universidad César Vallejo]. https://repositorio.ucv.edu.pe/bitstream/handle/20.500.12692/21547/Costilla_CPC.pdf?sequence=1 \&isAllowed=y

Cruz, C. (2017). Autoestima y nivel de desempeño docente de la universidad autónoma de Ica [Universidad Autónoma de Ica]. http://repositorio.autonomadeica.edu.pe/handle/autonomadeica/144

Ferrer, R. (2015). La influencia del factor humano, el liderazgo y la cultura de las organizaciones en los procesos de implementación y gestión del cambio organizacional. Rev. Int. Investig. Cienc. Soc., 11(1), 102-114. https://doi.org/http://dx.doi.org/10.18004/riics.2015.julio.102-114

Figueroa, L. (2015). Relación entre la cultura organizacional y el desempeño laboral de los colaboradores de una institución gubernamental [Universidad Rafael Landívar]. http://recursosbiblio.url.edu.gt/tesisjcem/2015/05/43/Figueroa-Leyvi.pdf

García, C. H. (2012). Bienestar Docente Y Pensamiento Emocional. Revista Fuentes, 12, 47-68. https://idus.us.es/handle/11441/32947

Glotova, G., \& Wilhelm, A. (2014). Teacher's Self-concept and Self-esteem in Pedagogical Communication. Procedia - Social and Behavioral Sciences, 132, 509-514. https://doi.org/10.1016/j.sbspro.2014.04.345

González Díaz, R. A., Ochoa Jiménez, S., \& Celaya Figueroa, R. (2016). Cultura organizacional y desempeño en instituciones de educación superior: implicaciones en las funciones sustantivas de formación, investigación y extensión. Universidad \& Empresa, 18(30), 13-31. https://doi.org/10.12804/rev.univ.empresa.30.2016.01

Hamui-Sutton, A., Vives-Varela, T., Gutiérrez-Barreto, S., Castro-Ramírez, S., Lavalle-Montalvo, C., \& Sánchez-Mendiola, M. (2014). Cultura organizacional y clima: el aprendizaje situado en las residencias médicas. Investigación En Educación Médica, 3(10), 74-84. https://doi.org/10.1016/s2007-5057(14)72730-9

Hernández, V. (2017). Las competencias emocionales del docente y su desempeño profesional. Alternativas En Psicología, 21(37), 79-92. http://bit.ly/2pjSzXt

Iglesias, A., \& Sánchez, Z. (2015). Generalidades del clima organizacional. Medisur, 13(3), 455-457. http://www.medisur.sld.cu/index.php/medisur/article/view/3047

Irigoyen Rivera, E., Llamo Guerrero, S., \& Pérez Silva, E. (2011). La Autoestima Y Su Relación Con El Clima Institucional En Los Docentes De Primaria De La I. E. No 00500 German Rojas Vela De Soritor - Moyobamba - 2011 [Universidad Cesar Vallejo]. https://repositorio.ucv.edu.pe/handle/20.500.12692/31543

Khezerlou, E. (2017). Professional self-esteem as a predictor of teacher burnout across Iranian and Turkish EFL teachers. Iranian Journal of Language Teaching Research, 5(1), 113-130. https://files.eric.ed.gov/fulltext/EJ1132488.pdf

Lacherre Calderón, E. (2017). Cultura Organizacional en los docentes de Zegel - IPAE, Piura 2016 [Universidad de Piura]. In Universidad de Piura. https://pirhua.udep.edu.pe/handle/11042/2989

Llancari Morales, R. A., \& Alania Contreras, R. D. (2020). Cultura organizacional y desempeño docente en la Universidad Nacional del Centro del Perú. Socialium, 4(1), 22-33. https://doi.org/https://doi.org/10.31876/sl.v4i1.45

Loli Pineda, A., \& Cuba B., E. (2007). Autoestima y compromiso organizacional en trabajadores de una universidad pública de provincias. Revista de Investigación En Psicología, 10(1), 103-108. https://doi.org/https://doi.org/10.15381/rinvp.v10i1.3910

Loyo De Falcón, R. (2015). La cultura organizacional desde la perspectiva del docente UNEFA. Omnia, 21(2), 53-70. https://www.redalyc.org/articulo.oa?id=73743366004

Martínez-Torres, S. (2020). Importancia del autoconcepto profesional docente desde la perspectiva de organismos internacional. Educación y Ciencia, 9(53), 129-136. http://www.educacionyciencia.org/index.php/educacionyciencia/article/view/552/456540

Moreno, G., \& Pineda, R. (2019). Determinación de relación entre cultura organizacional y desempeño docente. Revista Científica Hallazgos21, 4 4(2), https://revistas.pucese.edu.ec/hallazgos21/article/view/340

Naranjo, M. (2007). Autoestima: Un factor relevante en la vida de la persona y tema esencial del proceso educativo. Revista Electrónica “Actualidades Investigativas En Educación,” 7(3), 1-27. http://www.redalyc.org/articulo.oa?id=44770311

Pacheco, M. E. (2019). El clima organizacional y la autoestima en la Institución Educativa integrada $N^{\circ} 31756$ Ricardo Palma - La Esperanza- Chaupimarca - Pasco [Universidad Nacional de educación]. https://repositorio.une.edu.pe/handle/UNE/2862 
Panesso, K., \& Arango, M. (2017). La autoestima, proceso humano. Psyconex, 9(14), 1-14. https://aprendeenlinea.udea.edu.co/revistas/index.php/Psyconex/article/viewFile/328507/20785325

Peñaherrera León, M., Cachón Zagalaz, J., \& Ortiz Colón, A. (2014). La autoestima profesional docente y su implicación en el aula. Magister, 26(1), 52-58. https://doi.org/10.1016/s0212-6796(14)70018-0

Rahmadani, F., \& Hanum, F. (2020). Organizational culture. Advances in Social Science, Education and Humanities Research, 88-91. https://doi.org/10.1115/JRC2017-2247

Ramírez Campos, Á. F., \& Domínguez Aguirre, L. R. (2012). El clima organizacional y el compromiso institucional en las ies de puerto vallarta. Investigación Administrativa, 109, 21-30. https://doi.org/10.35426/iav41n109.02

Rodríguez Castellanos, C., \& Romo Rojas, L. (2013). Relación entre cultura y valores organizacionales. Conciencia Tecnológica, 45, 12-17. https://dialnet.unirioja.es/servlet/articulo?codigo=4425550

Romero, S. E. (2019). Cultura organizacional y desempeño en los docentes de una institución educativa en Ventanilla - Callao. http://repositorio.usil.edu.pe/handle/USIL/8803

Sadeghi, K., \& Sa'adatpourvahid, M. (2016). EFL teachers' stress and job satisfaction: What contribution can teacher education make? Iranian Journal of Language Teaching Research, 4(3), 75-96. https://files.eric.ed.gov/fulltext/EJ1127347.pdf

Sebastian, V. (2012). Autoestima y Autoconcepto docente. Phainomenon, 11(1), 23-34. http://www.unife.edu.pe/publicaciones/revistas/filosofia/Phainomenon/2012/articulo 2.pdf

Tabassum, F. (2012). Autoestima profesional de los profesores de secundaria. Ciencias Sociales Asiáticas, 8(2), 206-210. https://doi.org/http://dx.doi.org/10.5539/ass.v8n2p206

Tinoco Gómez, O., Quispe Atúncar, C., \& Beltrán Saravia, V. (2014). Cultura organizacional y satisfacción laboral en la facultad de Ingeniería Industrial en el marco de la acreditación universitaria. Industrial Data, 17(2), 56-66. https://doi.org/10.15381/idata.v17i2.12048

Valero-Ancco, V. N., Huaman-Huaman, L., \& Garavito-Checalla, E. C. (2021). Autoestima e identidad profesional de las profesoras de educación inicial. Investigación Valdizana, 15(1), 24-30. https://doi.org/10.33554/riv.15.1.808

Vasquéz Alatorre, A. (2013). Interdependencia entre el liderazgo transformacional, cultura organizacional y cambio educativo: una reflexión. Revista Iberoamericana Sobre Calidad, Eficacia y Cambio En Educación, 11(1), 73-91. https://www.redalyc.org/pdf/551/55125665006.pdf

Wilhelm, K., Martin, G., \& Miranda, C. (2012). Autoestima profesional: competencia mediadora en el marco de la evaluación docente. Revista Latinoamericana En Ciencias Sociales, Niñez y Juventud, 10(1), 339-350. $\quad$ http://revistaumanizales.cinde.org.co/rlcsnj/index.php/RevistaLatinoamericana/article/view/610

Zegarra, R., \& Velázquez, M. (2016). El coaching: una forma para fortalecer el profesionalismo del docente en el aula. Revista Páginas de Educación, 9(2), 1-34. https://doi.org/https://doi.org/10.22235/pe.v9i2.1294 